\title{
Membrane-based separation and concentration of total flavone glycosides from Desmodium styracifolium
}

\author{
Kun Yang, Ze-Bin Guo, Kun-Ping $\mathrm{Li}^{*}$ \\ Guangdong Pharmaceutical University, Guangzhou 510006, China
}

\begin{abstract}
Desmodium styracifolium is one of the traditional Chinese herbs. In the present study, membrane-based technologies were used to separate and concentrate of the total flavone glycosides fraction from D. styracifolium. The extracts flowed through an ultrafiltration membrane which MWCO (molecular weight cut-off) is $30 \mathrm{KDa}$ and the permeate was concentrated by MWCO-1KDa nano-filtration membrane. The solid content of the membrane concentrated extracts of D. styracifolium (MEDs) was $26.5 \mathrm{mg} / \mathrm{ml}$. Moreover, the content of vicenin-2, schaftoside and isovitexin in MEDs were $4.88 \%, 9.76 \%, 1.89 \%$ respectively. The assay in vitro showed MEDs has better anti-inflammatory effect which partly proved that our membrane-based processes for separation and concentration of flavone glycosides from $D$. styracifolium is reliable and practicable.
\end{abstract}

\section{Introduction}

D. styracifolium, the dry aerial part of the $D$. styracifolium (Osb.) Merr, is one of the traditional Chinese herbs grown in south China [1]. Numerous researches have reported that the flavone glycosides fraction of D. styracifolium for its anti-glycation [2], anti-apoptosis and autophagy [3], antioxidation [4, 5] anti-inflammatory [6] and attenuation of kidney injuries $[1,7,8]$ bioactivities. It is widely used in treatment of renal calculi, and it is also used for production of some Chinese patent drugs, such as Fu-Fang-Jin-Qian-Chao herbal granules [8] and Shilintong Tablet [9]. Therefore, how to separate and purify the flavonoids fraction in $D$. styracifolium efficiently has been a technical topic worthwhile to study.

In recent decade, membrane-based technologies have demonstrated to be an effective and clean way, which can be used for recovery, separation and concentration of natural product [10-12]. Membrane-based technologies are using a selective passable membrane as separation medium. The material passes or cut-off the molecular that depends on the pore size of the filter membrane to achieve the purpose of separation and purification [13]. At present, quite a few of studies were focused on the application of membrane technology in pharmaceuticals and natural products [10-16]. Zhao. etc [16] reported the membrane separation of flavonoids in Lotus Germ, but they didn't involve the membrane concentration of the extract and its effect on biological activity.

In this paper, an innovative workflow was proposed to separate and concentrate the flavone glycosides from D. styracifolium.

\section{Materials and methods}

\subsection{General description}

Shown as Figure 1, the total flavone glycosides were extracted from raw material by routinely water extraction, concentration, alcohol precipitation and filtration. Then through a microfiltration (MF) membrane with an average pore diameter of $0.2 \mu \mathrm{m}$. Subsequently, flowed through an ultrafiltration (UF) membrane which molecular weight cut-off (MWCO) is $30 \mathrm{KDa}$ and the permeate was concentrated by nanofiltration (NF) membrane of MWCO-1KDa. Finally, the product was dried by speed-vacuum drying.

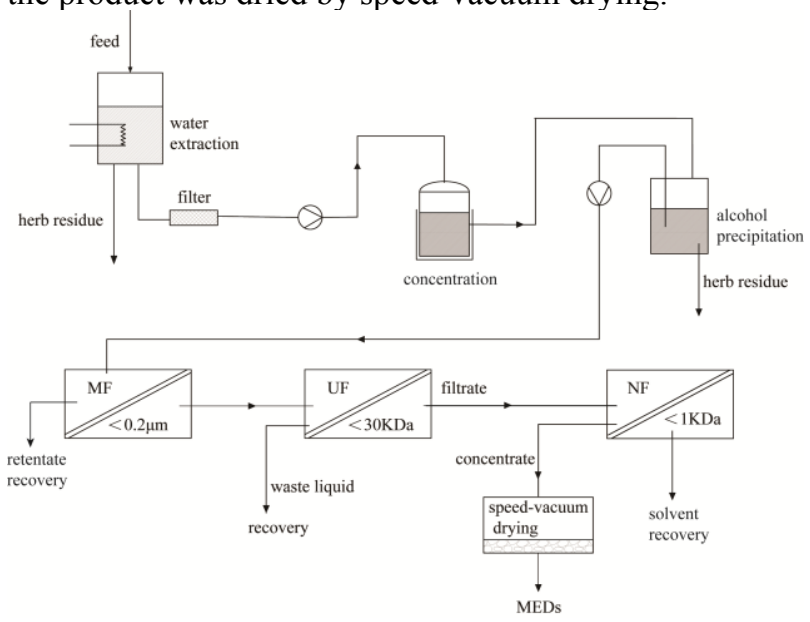

Fig. 1. The technological route of membrane-based separation and concentration of total flavone glycosides.

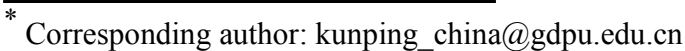


MF: microfiltration membrane UF: ultrafiltration membrane

NF nanofiltration membrane; MEDs: membrane concentrated extracts of $D$. styracifolium

\subsection{Microfiltration of crude extract solution}

According to the traditional technology [4], powdered dried D. styracifolium $(5 \mathrm{~kg})$ were extracted two times with water $(60 \mathrm{~L})$ at $95 \sim 98{ }^{\circ} \mathrm{C}$ for $2 \mathrm{~h}$. The extract was concentrated to $4.5 \mathrm{~L}$. Then the precipitated by adding alcohol to concentration of $65 \%$. After being stewed $24 \mathrm{~h}$, the mixture was filtered through the metal agglomeration filter core (50 60 m, Hengge Technology Co., Ltd., China) and a $0.2 \mu \mathrm{m}$ MF membrane (Tianjin Jintang Experimental Equipment Co., Ltd., China). In the end, we obtained the microfiltrate of $0.2 \mu \mathrm{m}$ MF membrane of D. styracifolium (Fig.2A).

\subsection{Ultrafiltration}

To remove the substances with a molecular weight greater than 30,000 from the microfiltrate, MWCO$30 \mathrm{KDa}$ UF membrane (Suez Environnement, French) was selected and loaded into the membrane separation experiment equipment (Hangzhou Science and technology membrane water treatment engineering Co., Ltd., China). The permeate of $0.2 \mu \mathrm{m}$ microfiltrate was purified through the system. Finally, we get permeate of MWCO-30KDa UF membrane (Fig.2B).

\subsection{Concentration with nanofiltration}

The permeate of MWCO-30KDa UF membrane obtained was concentrated with MWCO-1KDa NF membrane (Suez Environnement, French), and the membrane permeate (Fig.2C) and retentate (Fig.2D) were obtained.

\subsection{Speed-vacuum concentration}

The specimens were respectively sampled $1 \mathrm{ml}$ triply from the $0.2 \mu \mathrm{m}$ microfiltrate of $D$. styracifolium and the MWCO-1KDa NF membrane permeate and retentate. Then, these specimens were dried by speed-vacuum concentrated in vacuum $(6.2 \mathrm{mbar})$ at $50^{\circ} \mathrm{C}$ and 4300r/min (Rvc 2-25 CD plus, CHRIST, Germany).

\subsection{Qualitative and quantitative analysis of EDs and MEDs}

Vicenin-2, schaftoside and isovitexin were three main components in D. styracifolium according to the previous reports [17,18]. Qualitative analysis of permeate of MWCO-30KDa UF membrane were performed by liquid chromatograph-mass spectrometer (LC-MS) (1290 UHPLC-6545 QTOF-MS, Agilent Corporation, CA, USA) with LC column (ZORBAX RRHD SB-C18, $2.1 \times 100 \mathrm{~mm}, 1.8 \mu \mathrm{m}$, Agilent Corporation, CA, USA). The mobile phase was acetonitrile-phosphoric acid-water with gradient elution. In detail, the flow rate of mobile phase was $0.2 \mathrm{~mL} / \mathrm{min}$.
The wavelength of detection, the injection volume and the column temperature were $280 \mathrm{~nm}, 2 \mu \mathrm{l}$ and $35^{\circ} \mathrm{C}$ respectively. Structures were identified by the data acquisition at $\mathrm{m} / \mathrm{z} 593,563$, and 431 and the retention time.

Quantitative analysis of three flavone glycosides was carried on High-Performance Liquid Chromatography (HPLC) (LC-20, Shimadzu, Japan) with C18 column $(4.6 \times 250 \mathrm{~mm}$, Kromasil, Switzerland). of samples were loaded through Using gradient elution with acetonitrilephosphoric acid-water as the mobile phase, $280 \mathrm{~nm}$ as detected wavelength, and injection volume as 10 microliter. To protract standard curve, linear regression analysis was made by the peak area $(\mathrm{Y})$ and the and the reference substances mass $(\mathrm{X})$. The injection volume of mixed three reference substances was $5,10,15,20 \mu \mathrm{l}$.

\subsection{Anti-inflammatory effect assay in vitro}

To investigate and compare the anti-inflammatory effect of flavonoid content of extraction from different method, RAW 264.7 cells were incubated with EDs (extracts of D. styracifolium) and MEDs (membrane concentrated extracts of D. styracifolium) at the concentration of $80 \mathrm{~g} / \mathrm{ml}$ for $2 \mathrm{~h}$, followed by stimulation with lipopolysacchride (LPS) (1 g/ml) for $24 \mathrm{~h}$. Then, mRNA expression level and pro-inflammatory cytokines including tumor necrosis factor- $\alpha$ (TNF- $\alpha)$ and interleukin-6 (IL-6) were analysed by RT-qPCR.

\section{Results and discussion}

\subsection{Concentration with nanofiltration membrane}

By using 0.2 $\mu \mathrm{m}$ MF membrane, MWCO-30,000 UF membrane and the MWCO-1,000 NF membrane in succession, EDs were isolated and purified. The comparison of apparent effects of EDs and MEDs were shown as Figure 2. The apprent of the extract obtained by the traditional technology is dark brown and the extract is turbid. As a comparison, the permeate of MWCO-30,000 UF membrane is light yellow and clear.

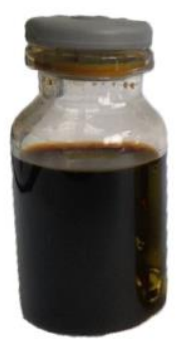

A

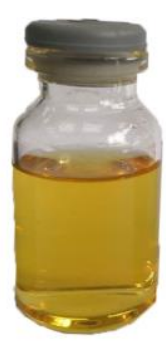

B

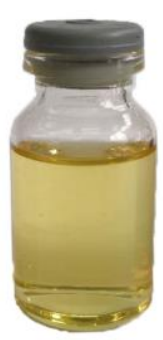

C

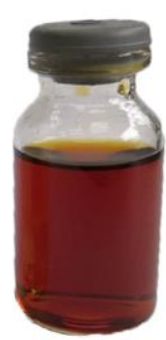

$\mathrm{D}$
Fig. 2. Comparison of apparent effects of EDs and different MEDs. (A) The permeate of $0.2 \mu \mathrm{m}$ MF membrane. (B)The permeate of MWCO-30KDa UF membrane. (C) The permeate of MWCO-1KDa NF membrane. (D) The retentate of MWCO$1 \mathrm{KDa}$ NF membrane (10 folds). 


\subsection{The content of total flavonoids of different membranes separation}

According to the HPLC results (Fig.3), the flavonoids in the microfiltrate of $0.2 \mu \mathrm{m}$ MF membrane is less than the permeate of MWCO-30KDa UF membrane and is very little in the MWCO-1KDa NF membrane permeate. The $0.2 \mu \mathrm{m}$ MF membrane can remove macromolecules such as suspended solids, carbohydrates (sugars), proteins, pectins, fibers ( $\beta$-glucan) [12]. The UF membrane can remove mycelium, virus and heat source. On the other hand, it can separate, concentrate, purify and recovery the macromolecule solution [13]. In the purpose of concentration, inorganic salt, organic acids, small molecules, metal ions and water can pass through the membrane and the solvent and solute in the extraction can be separated in the process of NF. In addition, there is no phase transformation in the process of concentration $[11,16]$. The solid concent of $0.2 \mu \mathrm{m} \mathrm{MF}$ membrane, MWCO-1,000 NF and MWCO-1,000 NF membrane permeate were $22 \mathrm{mg} / \mathrm{ml}, 26.5 \mathrm{mg} / \mathrm{ml}$ and $8 \mathrm{mg} / \mathrm{ml}$ respectively.
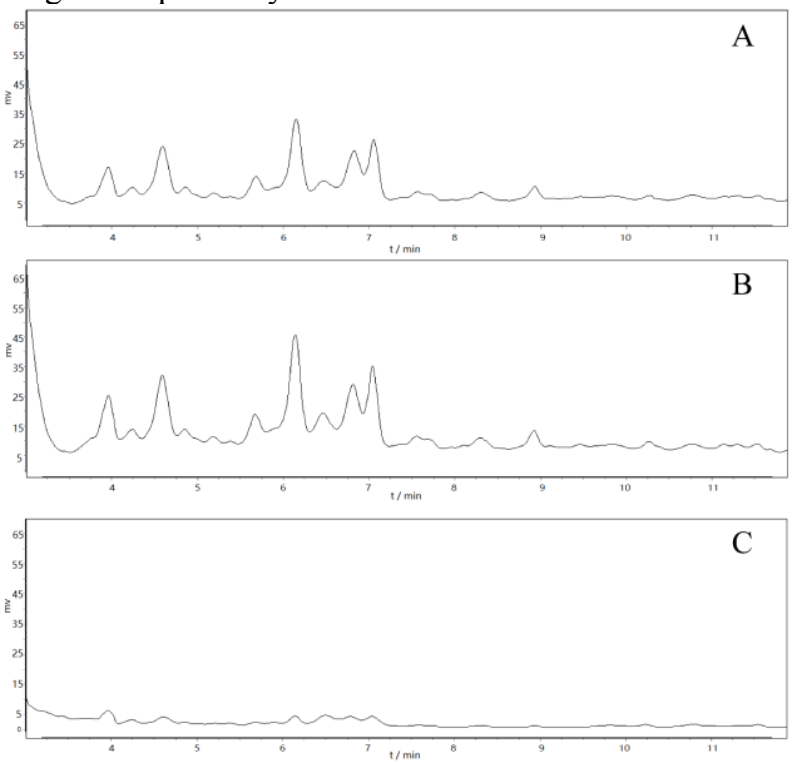

Fig. 3. Representative HPLC of total flavonoids with different membranes separation. (A) The microfiltrate of $0.2 \mu \mathrm{m} \mathrm{MF}$ membrane. (B) The MWCO-1,000 NF membrane. (C) The MWCO-1,000 NF membrane permeate.

\subsection{Qualitative analysis of three flavonoids in $D$. styracifolium by LC-MS}

To confirm that the flavone glycosides in MEDs had not been removed. The typical ion peaks for vicenin- 2 and schaftoside at $\mathrm{m} / \mathrm{z} 593$ and 563 , and isovitexin at $\mathrm{m} / \mathrm{z}$ 431 respectively extracted from the TIC spectra of the membrane retentate (Fig.4). In vicenin-2 msms, the ion peak of 563 is the whole molecule, its mass spectra has obvious peak of $563,473,297 \mathrm{~m} / \mathrm{z}$, respectively. In schaftoside msms, its mass spectra has obvious peak of $563,473,443,297 \mathrm{~m} / \mathrm{z}$, respectively. Isovitexin relative molecular weight is 431 , other fragments are 341,311 , $283 \mathrm{~m} / \mathrm{z}$, respectively. They are in consistent with the previous reasarches $[18,20]$.
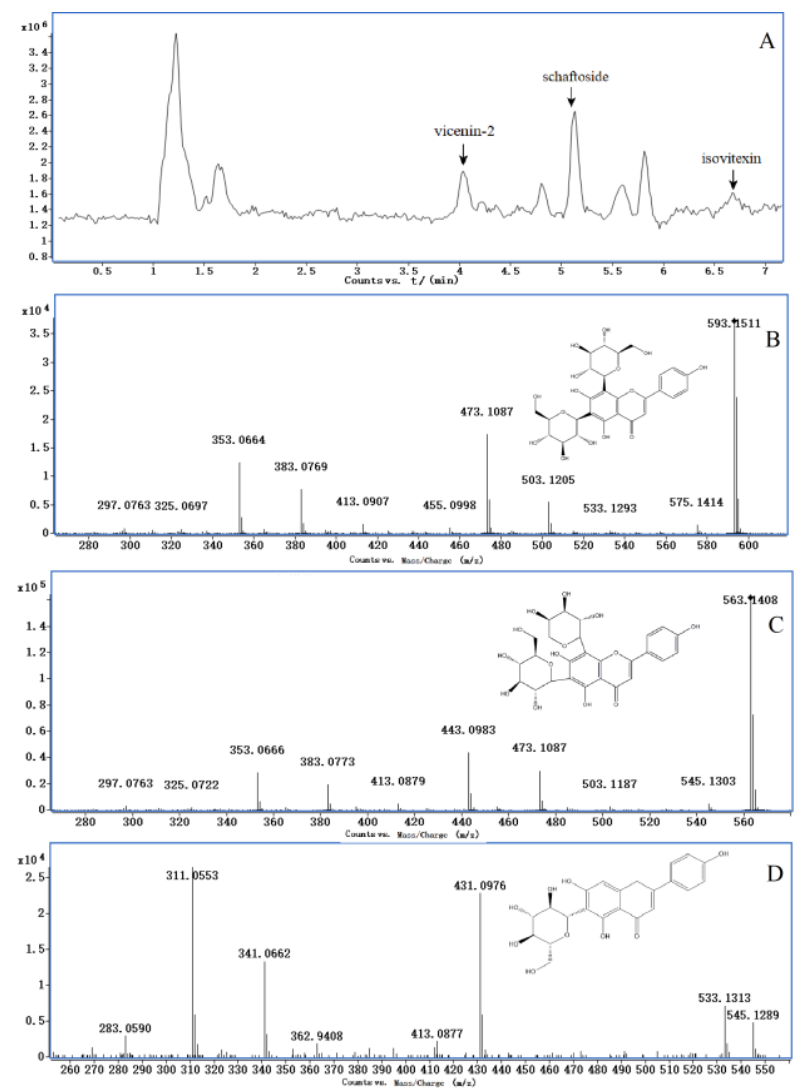

Fig. 4. Qualitative analysis of three flavonoids in $D$. styracifolium by LC-MS. (A) The MWCO- $1 \mathrm{KDa}$ NF membrane retentate. (B) Vicenin-2 in membrane retentate. (C) Schaftoside in membrane retentate. (D) Isovitexin in membrane retentate.

\subsection{Quantitative analysis of three flavone glycosides in EDs and MEDs by HPLC}

The regression equations, linear range and regression coefficient are shown in Table 1. As shown in Fig.5, these three flavone glycosides in EDs and MEDs could be separated well. The content of three flavone glycosides in MEDs are $4.88 \%, 9.76 \%, 1.89 \%$ respectively. The content was calculated by the following formula:

\section{Content $\%=$ (weight of the reference substance) $/$ (weight of the solid) $\times 100 \%$}

Table 1. Linear Relationship between three flavone glycosides in D. styracifolium.

\begin{tabular}{|c|c|c|c|}
\hline Compounds & $\begin{array}{c}\text { Regression } \\
\text { Equation }\end{array}$ & $\mathbf{r}$ & $\begin{array}{c}\text { Linear } \\
\text { Range/ } \mu \mathrm{g}\end{array}$ \\
\hline vicenin-2 & $\begin{array}{c}\mathrm{y}=4220320 \mathrm{x}- \\
1951\end{array}$ & 0.9935 & $\begin{array}{c}0.0178 \sim \\
0.0712\end{array}$ \\
\hline schaftoside & $\begin{array}{c}y=3664829 x- \\
2334\end{array}$ & 0.9984 & $\begin{array}{c}0.017 \sim \\
0.068 \\
\end{array}$ \\
\hline isovitexin & $\begin{array}{c}\mathrm{y}=3412697 \mathrm{x}- \\
1944\end{array}$ & 0.9961 & $\begin{array}{c}0.0117 \sim \\
0.0468\end{array}$ \\
\hline
\end{tabular}




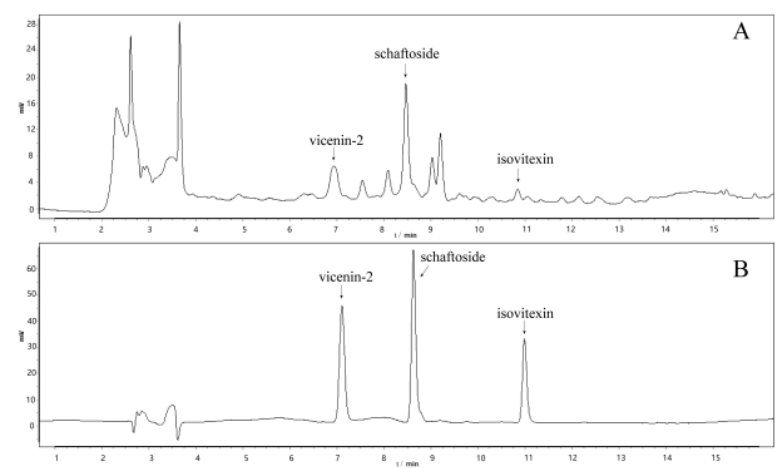

Fig. 5. HPLC of the MWCO-1,000 NF membrane permeate and reference substance. (A) The MWCO-1,000 NF membrane retentate. (B) Reference substance of vicenin-2, schaftoside and isovitexin.

\subsection{Anti-inflammatory effect of Eds and MEDs on RAW 264.7 cells}

LPS, derived from the cell wall of gram-negative bacteria, can induce a sepsis syndrome accompanied [1]. In this experiment, LPS was used to induce inflammation response in RAW 264.7 cells through promoting the release of various pro-inflammatory factors. TNF- $\alpha$, IL- $1 \beta$ and IL- 6 are all pro-inflammatory cytokines in NF- $\kappa \mathrm{B}$ signaling pathway[1]. These cytokines are important in the generation of inflammation. The levels of the cytokines of TNF- $\alpha$ and IL-6 in RAW264.7 cells were significantly decreased by incubated with EDs and MEDs. And the decrease of MEDs was even more significant (Fig.6). That means MEDs has a better anti-inflammatory activity.
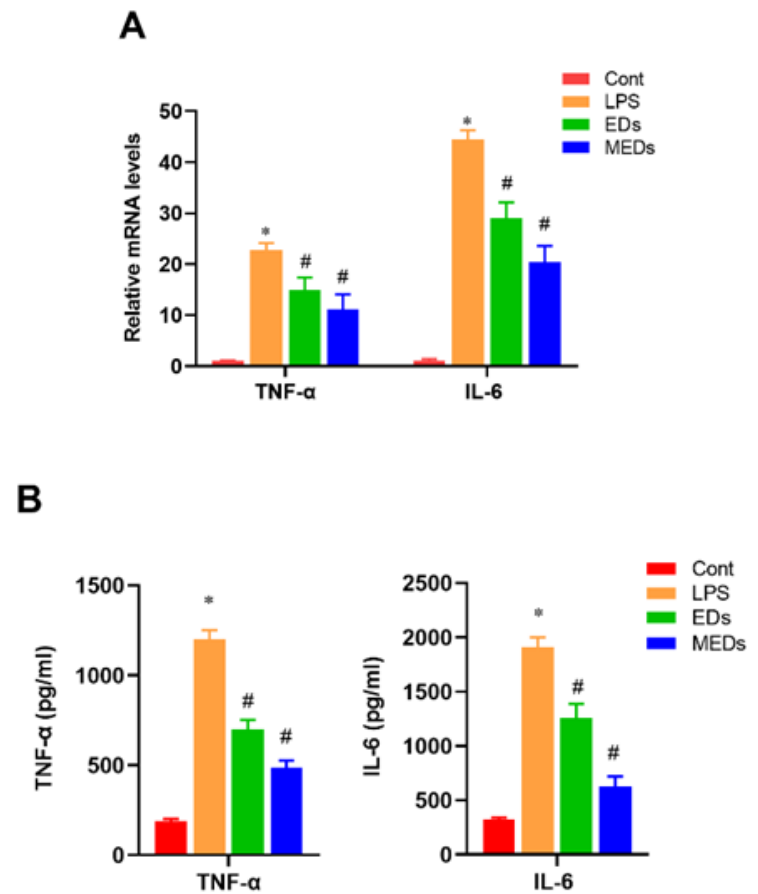

Fig. 6. Membrane concentrated extracts of D. styracifolium attenuated inflammation induced by LPS in macrophages. (A) MEDs down-regulated the relative mRNA expression levels of tumor necrosis factor- $\alpha$ (TNF- $\alpha$ ) and Interleukin-6 (IL-6) induced by LPS (1ug/ml) in macrophages. (B) MEDs obviously inhibited pro-inflammatory cytokines level, including TNF- $\alpha$ and IL- 6 , induced by LPS (1ug/ml) in macrophages. Data are presented as mean $\pm \mathrm{SD}(\mathrm{n}=6),{ }^{*} \mathrm{p}$ $<0.05$ vs. con group; $\# p<<0.05$ vs. LPS treated group.

\section{Conclusions}

In this study, we provided an innovation of the technological route on the separation and concentration of flavonoids. After a series of separation and concentration of MF, UF and NF membranes, the total flavonoids were obtained with improved purity, compared with the traditional process.

As all results shown, the membrane-based separation method can separate active components through a sieving way based on their molecular weight [12]. The herb liquor after membrane-based separation and concentration is best characterized by its clearer apparent and higher content of total flavonoids than traditional process. Based on the test in vitro, the flavone glycosides after membrane-based separation and concentration has potentical to be developed as a more effective way to obtain flavone glycosides.

Our findings present innovative workflow for membrane-based separation and concentration of flavone glycosides.

\section{References}

1. J. Zhou, J. Jin, X. Li, Z. Zhao, L. Zhang, Q. Wang, J. Li, Q. Zhang,S. Xiang, Urolithiasis 46, 3, 231-241 (2018)

2. M. N. Islam, I. J. Ishita, H. A. Jung,J. S. Choi, Food Chem Toxicol 69, 55-62 (2014)

3. H. Xie, J. Li, H. Gao, J. Wang, C. Li, Y. Xu,C. Liu, Mol Cell Biochem 442, 1-2, 169-175 (2018)

4. Z. Zhang, Q. Zhong, B. Zhou, Z. Zhu, Y. Zhang, Y. Wei, Y. Chen, Chinese Medicine Modern Distance Education of China 17, 107-110 (2019)

5. L. Yu, J. Wang, S. Huang, B. Tan, Chinese Wild Plant Resources 33, 26-28 (2014)

6. J. Wang, Y. T. Liu, L. Xiao, L. Zhu, Q. Wang,T. Yan, Inflammation 37, 6, 2085-2090 (2014)

7. Y. Liu, Z. Wang, D. Yang, X. Liu, X. Gao, X. Hui, Chin J Gernoto 38,10, 2467-2470, (2018)

8. W. Chen, W. Liu, J. Hou, J. Ding, Z. Peng, S. Gao, X. Dong, J. Ma, Q. Lin, J. Lu, Z. Guo, Biosci Rep 39, 2, (2019)

9. H.LIU, H. Li, J China Pharm 27, 12, 1710-1713 (2016)

10. H. Tang, J. Yang, K. Gu, Y. Mu, Q. Wu, Z. Huang, SCI TECHNOL FOOD IND, (2019)

11. R. Castro-Muñoz,V. Fíla, Trends in Food Science \& Technology 82, 8-20 (2018)

12. R. Castro-Muñoz, J. Yáñez-Fernández, V. Fíla, Food Chem 213, 753-762 (2016)

13. F.Wu, Tianjian Pharm 22, 2, 65-69(2010) 
14. R. Castro-Munoz, C. Conidi,A. Cassano, Crit Rev Food Sci Nutr 59, 18, 2927-2948 (2019)

15. A. Cassano, C. Conidi, R. Ruby-Figueroa,R. CastroMunoz, Int J Mol Sci 19, 2, (2018)

16. W. Zhao, H. Li, S. Pan, X. Xu, CIFST 17, 8, 165169 (2017)

17. L. Chen, X. Cheng, X. Tang, Q. Yang, Chin J Chin Mater Med 43, 16, 3322-3328 (2018)

18. X. Liu, X. Fan, X. Wang, R. Liu, C. Meng,C. Wang, J Pharm Biomed Anal 171, 52-64 (2019)

19. X.Yang, Y. LI, L. LI, M. LIU,H. Qian, Chin Tradit Herbal Drugs 50, 1, 134-141 (2019)

20. K. Li, Z. He, X. Wang, M. Pineda, R. Chen, H. Liu, K. Ma, H. Shen, C. Wu, N. Huang, T. Pan, Y. Liu,J. Guo, Free Radic Biol Med 124, 163-175 (2018) 